\title{
Effects of Transitional Care on Hospital Readmission and Mortality Rate in Subjects With COPD: A Systematic Review and Meta-Analysis
}

\author{
Edi Sampurno Ridwan, Hamam Hadi, Yu-Lin Wu, and Pei-Shan Tsai
}

\begin{abstract}
BACKGROUND: Studies on the effect of transitional care on hospital readmissions have reported inconsistent findings, and the effect on mortality has not been reviewed systematically. This systematic review and meta-analysis of randomized controlled trials aims to examine the effect of transitional care interventions on COPD-related readmissions, all-cause hospital readmissions, and all-cause mortality rates in subjects with COPD. METHODS: Electronic databases (CINAHL, Embase, Scopus, MEDLINE, Cochrane, PubMed, Web of Science, Airity, BMJ Respiratory Research Journal, and National Digital Library of Theses and Dissertations) were searched from inception to April 26, 2017. Online searches were conducted using key words and MeSH terms for COPD and transitional care. Entry terms for searching included chronic obstructive pulmonary disease, COPD, COPD transitional care or care transition, continuity of patient care, patient discharge, and patient transfer. The quality of the included trials was assessed using the Cochrane Collaboration tool. RESULTS: 13 randomized controlled trials met the inclusion criteria. Transitional care significantly reduced the risk of COPD-related readmissions (odds ratio $=0.599,95 \%$ CI $0.421-0.852$ ) and all-cause hospital readmissions (odds ratio $=0.720,95 \%$ CI $0.531-0.978$ ), but not that of all-cause mortality (odds ratio $=0.863,95 \%$ CI $0.576-1.294$ ) in subjects with COPD. The effects of transitional care on hospital readmissions were moderated by the duration of interventions, type of care providers, and use of telephone follow-up as an element of the intervention. CONCLUSIONS: There was a significant effect of transitional care on both COPD-related and all-cause hospital readmissions in subjects with COPD. Duration of interventions, type of care providers, and use of telephone follow-up appeared to moderate the beneficial effects of transitional care. Key words: COPD; continuity of patient care; patient discharge; patient transfer; transitional care; transition care. [Respir Care 2019;64(9):1146-1156. () 2019 Daedalus Enterprises]
\end{abstract}

\section{Introduction}

COPD affects 210 million people globally and accounts for 4 million deaths annually. In 2002, it was the fifth most

\footnotetext{
Mr Ridwan is affiliated with the School of Nursing, College of Nursing, Taipei Medical University, Taipei, Taiwan, and with the School of Nursing, Faculty of Health Sciences, Alma Ata University, Yogyakarta, Indonesia. Dr Hadi is affiliated with the Department of Nutrition, Faculty of Health Sciences, Alma Ata University, Yogyakarta, Indonesia. Dr Wu is affiliated with the Department of Nursing, St Mary's Junior College of Medicine, Nursing, and Management. Dr Tsai is affiliated with the School of Nursing, College of Nursing, Taipei Medical University, Taipei, Taiwan; with the Department of Nursing and Research Center of Big Data and Meta-analysis, Wan Fang Hospital, Taipei Medical University, Taipei, Taiwan; and with the Sleep Science Center, Taipei Medical University, Taipei, Taiwan.
}

common cause of death, and it is projected to become the fourth most common cause of mortality by $2030 .{ }^{1}$ COPD is a common, preventable, and treatable disease with characteristics including persistent respiratory symptoms and chronic air-flow limitation caused by airway narrowing or

\footnotetext{
Mr Ridwan has disclosed a scholarship provided by the Indonesia Endowment Fund for Education and the Ministry of Research, Technology, and Higher Education. The other authors have disclosed no conflicts of interest.

Correspondence: Pei-Shan Tsai PhD, School of Nursing, College of Nursing, Taipei Medical University, $250 \mathrm{Wu}$ Xing Street, Taipei, Taiwan. E-mail: ptsai@tmu.edu.tw.
}

DOI: $10.4187 /$ respcare.06959 
alveolar abnormalities as a result of cumulative exposure to harmful gasses and particles combined with host factors. ${ }^{2}$ The pathological characteristics of COPD include irreversible air-flow obstruction and abnormal inflammatory responses in the lungs that represent innate and adaptive immune responses to long exposure to toxic agents. This response may lead to hypersecretion of mucus, tissue destruction, and disruption of normal repair and defense mechanisms, all of which result in inflammation of small airways and fibrosis. ${ }^{3}$ When advanced, these responses can cause pulmonary hypertension, cor pulmonale, and left heart dysfunction. ${ }^{4,5}$ Thus, morbidity from COPD may be directly related to physiological defects and can be concomitant with other chronic conditions. Furthermore, the worsening of pulmonary inflammation in COPD might cause frequent disease relapse, prolonging physiological recovery and leading to repeated acute care demands. ${ }^{6,7}$ High COPD exacerbation incidence strongly corresponds to high morbidity, mortality, and hospital readmission rates; high frequency of emergency visits; worsened health-related quality of life; and high costs. - $^{6-11}$

These issues exacerbate various burdens on individual patients, families, and the health care system. Patients with COPD discharged without home care are likely to be readmitted. ${ }^{12}$ Approximately $24 \%$ and $43 \%$ patients with COPD are readmitted at least once for any reason within $30 \mathrm{~d}$ and $90 \mathrm{~d}$ after discharge, respectively. ${ }^{13}$ The risk factors for rehospitalization in patients with COPD vary depending on the patient's conditions, ${ }^{14,15}$ care provider, and system factors. ${ }^{14}$ Other risk factors for rehospitalization include old age at diagnosis, COPD severity, current smoking habit, patient deprivation, intervention before admission, low body mass index, and comorbidities. ${ }^{14,16}$ Among these factors, 2 modifiable risk dynamics of early readmission for patients with COPD are provider and system aspects. ${ }^{14}$ When the readmission rate is high and unexplained, it reflects problems in the transition process and post-discharge management. ${ }^{17}$ Health care providers in acute care settings should carefully review the discharge process to mitigate readmission risks. ${ }^{13}$ Furthermore, because evidence indicates a correlation between the readmission rate and mortality rate, health care services should ensure proper comorbidity management and resource allocation to identify, review, and increase management for patients with COPD at particular risk of hospital readmission. ${ }^{18}$ Evidence suggests that patients with COPD readmitted for a comorbid condition, such as infarction, pneumonia, septicemia, or pulmonary heart disease, are more likely to die within $30 \mathrm{~d}$ than are those readmitted with COPD alone. ${ }^{19}$ Properly managed and effective care transitions for patients with COPD can mitigate the risk of readmission and mortality, particularly for those who require complex care from a wide range of services.
Transitional care has been adopted by some health systems as a discharge intervention for patients with chronic conditions. Transitional care refers to a time-limited intervention designated to promote safety and timely transfer of patients between levels of health care or across care settings. ${ }^{20,21}$ Evidence indicated that transitional care components varied among the existing models, which mostly included predischarge and postdischarge elements and were delivered by a multidisciplinary team. ${ }^{20,22}$ However, transitional care for patients with COPD remains challenging due to the complexity of disease conditions. Although a meta-analysis showed effectiveness of transitional care in reducing hospital readmission rates in subjects with COPD at $>6$ and 18 months after discharge, ${ }^{23}$ this effect was found in a meta-analysis involving only a few studies including papers published from 1999 to 2013. With such a small number of analyzed trials, findings of the effectiveness of transitional care on readmission have been inconclusive. Through searching more databases, this metaanalysis was conducted to provide updated evidence on the effect of transitional care for subjects with COPD in order to inform clinical practice. Moreover, the effect of transitional care interventions on mortality has not been examined systematically. To elucidate this effect, this metaanalysis examined the effect of transitional care interventions on hospital readmissions and mortality rates in adults with COPD. Additional analyses were performed to identify potential moderators of the effects of transitional care on outcomes and to identify sources of heterogeneity across studies, if any, by analyzing covariates in moderator analyses.

\section{Methods}

This meta-analysis included randomized controlled trials with participants who were $\geq 18$ y old and diagnosed with COPD. Trials were included if the study utilized transitional care interventions prior to hospital discharge, or after hospital discharge, or if the study used bridging programs (Fig. 1). We compared a transitional care intervention with any form of nontransitional care, defined by primary studies as conventional care, usual care, standard care, or no treatment. This analysis included studies that reported COPD-related readmissions, all-cause hospital readmissions, COPD-specific mortality, and all-cause mortality as study outcomes.

The Preferred Reporting Items for Systematic Review and Meta-Analysis ${ }^{24}$ was adopted to specify objectives and methods of this meta-analysis. Ten electronic databases (ie, CINAHL, Embase, Scopus, MEDLINE, Cochrane, PubMed, Web of Science, Airity, BMJ Respiratory Research Journal, and the National Digital Library of Theses and Dissertations) were searched with no limit on language, date of publication, or outcomes for records until 


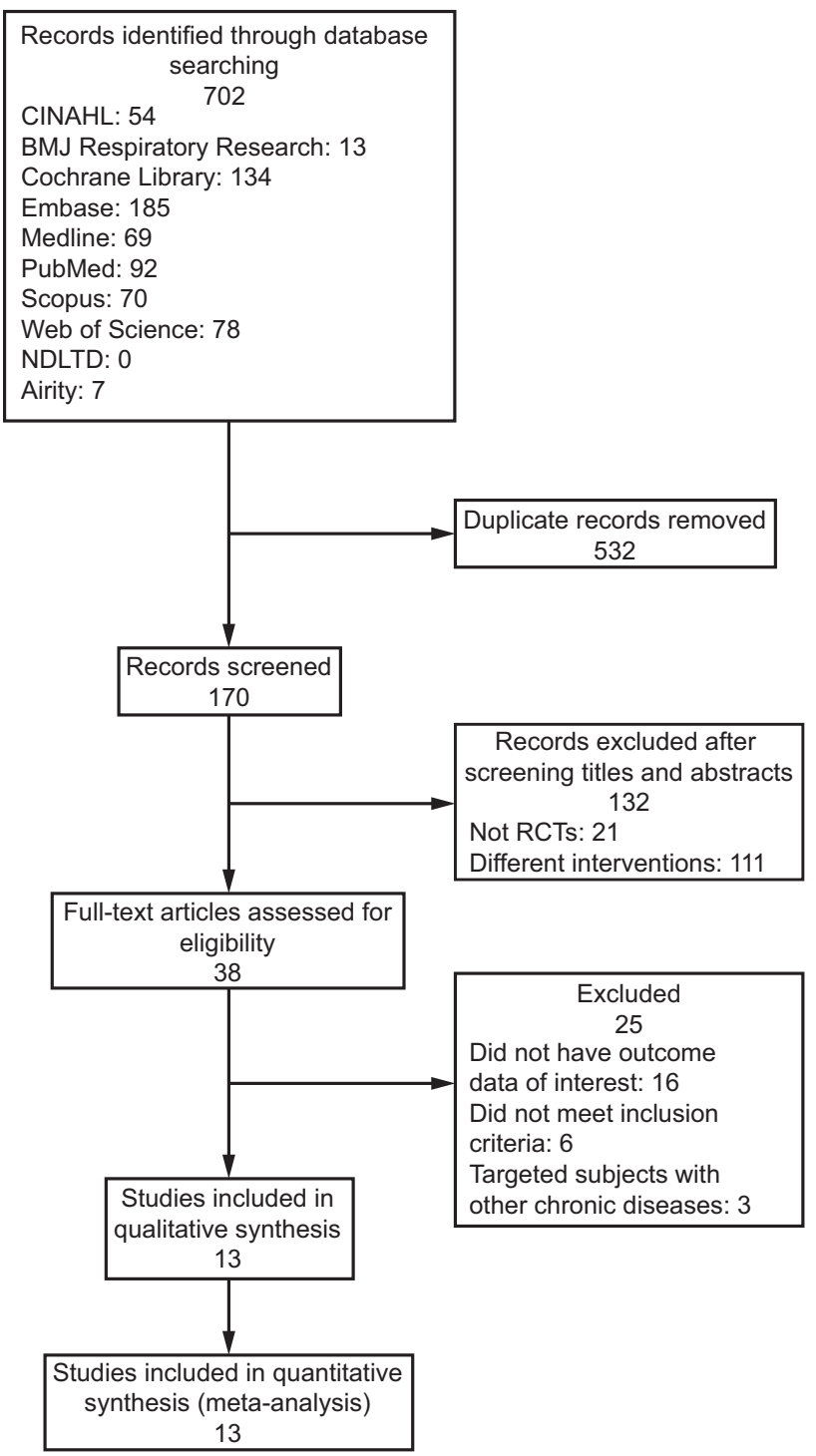

Fig. 1. Flow chart. RCT $=$ randomized controlled trial.

April 26, 2017. MeSH terms used as key words for searching articles encompassed chronic obstructive pulmonary disease, COPD, COPD transitional care or care transition, continuity of patient care, patient discharge, and patient transfer. Reference lists of the included trials were screened to identify relevant articles for this study that could have been missed during the article search.

All identified duplicate papers were removed. A 2-step trial selection was then performed by 2 reviewers [ESR and YLW]. First, the titles and abstracts were screened independently by the 2 reviewers. Second, full texts of the potential eligible articles were retrieved and reviewed by the reviewers independently based on the eligibility criteria. Consensus was reached by the 2 reviewers on the eligible references to be included for further synthesis and analysis.
We performed the methodology quality assessment. The quality of the study for individually included trials was assessed independently by the 2 reviewers using the Cochrane Collaboration tool for assessing risk of bias in randomized trials, as summarized in Table 1. Each reviewer made impartial determinations of the risk of bias of each study, focusing on random-sequence generation, allocation concealment, blinding of participants, health care personnel, outcome measurement, data collectors, outcome assessors, and any reported bias. The 2 reviewers discussed to reach consensus on any disagreements before the result was presented to the third reviewer for final decisions.

Data were extracted independently by the 2 reviewers; the required modifications were made based on reviewer agreement. Any disagreements were resolved through discussion to find consensus. Information extracted from individual trials included participants' characteristics, study design, interventions, and outcome measures. All outcomes of interest at the final follow-up were extracted.

\section{Statistical Analysis}

Effect size of transitional care interventions was measured as odds ratios (ORs) with $95 \%$ CIs by using randomeffect models due to differentiation of true treatment effects in each study. Heterogeneity across included trials was assessed using the $Q$ test and $I^{2}$ statistics. A $Q \geq 0.05$ and $I^{2}<50 \%$ were considered homogeneous between trials, whereas substantial heterogeneity was defined as $Q<0.05$ and $I^{2}>50 \% .{ }^{25}$ We performed the Begg rank correlation test and the Egger intercept test with a significance level of 0.05 to assess for publication bias in all included studies. A funnel plot was also created as a visual tool to determine publication bias. ${ }^{26}$ Moderator and meta regression analyses were performed to identify sources of heterogeneity and potential moderators of the relationship of transitional care with readmission and mortality. Data were analyzed with Comprehensive Meta-Analysis Software 2.0 (BioStat, Edgewood, New Jersey).

\section{Results}

\section{Identification of the Trials}

Searches of the 10 electronic journal databases identified 702 articles. After removing duplicate articles, we screened titles and abstracts of 170 articles for eligibility. Studies were discarded if they provided interventions other than transitional care for subjects with COPD or were not randomized controlled trials. For further assessment, 38 full-text studies were retrieved. We excluded studies that did not have outcome data of interest, ${ }^{16}$ did not meet inclusion criteria, ${ }^{6}$ and addressed transitional care in COPD 
Transitional Care, Readmissions, and Mortality in COPD

Table 1. Risk of Bias Summary of Methodological Quality for Each Included Trial

\begin{tabular}{|c|c|c|c|c|c|c|c|}
\hline \multirow[b]{2}{*}{ Author, year } & \multicolumn{2}{|c|}{ Selective Bias } & \multirow[b]{2}{*}{$\begin{array}{l}\text { Performance } \\
\text { Bias* }\end{array}$} & \multicolumn{2}{|c|}{ Detection Bias } & \multirow[b]{2}{*}{$\begin{array}{c}\text { Attrition } \\
\text { Bias } \dagger\end{array}$} & \multirow[b]{2}{*}{$\begin{array}{c}\text { Reporting } \\
\text { Bias } \ddagger\end{array}$} \\
\hline & $\begin{array}{l}\text { Random Sequence } \\
\text { Generation }\end{array}$ & $\begin{array}{c}\text { Allocation } \\
\text { Concealment }\end{array}$ & & $\begin{array}{l}\text { Blinding of Outcome } \\
\text { Assessment (patient- } \\
\text { reported outcome) }\end{array}$ & $\begin{array}{l}\text { Blinding of Outcome } \\
\text { Assessment } \\
\text { (objective outcome) }\end{array}$ & & \\
\hline Benzo et al 2016 & + & - & - & + & + & + & $?$ \\
\hline Casas 2006 & + & - & $?$ & $?$ & + & + & $?$ \\
\hline Cotton et al 2000 & + & - & - & - & $?$ & + & - \\
\hline Eaton et al 2009 & + & + & + & $?$ & $?$ & + & + \\
\hline Hermiz et al 2002 & $?$ & - & - & - & - & + & - \\
\hline Jennings et al 2015 & + & $?$ & - & $?$ & - & + & - \\
\hline Johnson-Warrington et al 2016 & + & + & $?$ & + & $?$ & + & + \\
\hline Kwok et al 2004 & $?$ & + & $?$ & - & $?$ & + & $?$ \\
\hline Skwarska et al 2000 & + & - & - & - & $?$ & $?$ & - \\
\hline Utens et al 2012 & + & + & $?$ & $?$ & $?$ & + & $?$ \\
\hline Vianello et al 2016 & + & + & - & - & $?$ & + & $?$ \\
\hline Wong et al 2005 & + & + & $?$ & - & $?$ & + & $?$ \\
\hline Ko et al 2017 & + & + & $?$ & - & $?$ & + & $?$ \\
\hline $\begin{array}{l}\text { * ie, blinding of participants and personnel. } \\
\dagger \text { ie, incomplete outcome data. } \\
\text { †ie, selective reporting. } \\
+=\text { low risk of bias } \\
-=\text { high risk of bias } \\
?=\text { uncertain risk of bias }\end{array}$ & & & & & & & \\
\hline
\end{tabular}

and other diseases. ${ }^{3}$ We retained 13 trials that met the inclusion criteria for quantitative meta-analysis.

\section{Study Characteristics}

The characteristics of the 13 included trials are summarized in Table 2. In total, 2,029 subjects diagnosed with COPD were included. The mean age of trial participants was 69.9 y (range 64.4-76.4 y); 60\% of the participants were male. Of those included in the trials, $69.2 \%$ were categorized as having severe COPD with average predicted value of $\mathrm{FEV}_{1}=42.2 \%$ (36\%-47.6\%). Severe COPD was identified in 9 studies. ${ }^{27-35}$ The subjects' comorbid index at baseline was addressed in 5 trials. ${ }^{27,28,30,34,36}$ Nine trials 28,30-37 included active-smoker subjects. Most included trials had 2 arms divided into transitional care and usual care groups, with 1,096 (54\%) and 933 (45\%) subjects, respectively. In terms of geographical locations, 2 studies $(15.3 \%)$ studies were conducted in the United States, $6(46.1 \%)$ in European countries, and 5 trials $(38.4 \%)$ in the Asia-Pacific region. In terms of outcomes, one study presented both COPD-related and all-cause hospital readmissions as outcomes. Nine studies only had the data on all-cause hospital readmissions, three had only the outcome on COPD-related readmissions, and one study had both data all-cause and COPD-related readmissions. 6 studies presented outcome data on all-cause mortality, and 1 study had data of COPD-specific mortality.
The 13 trials varied in elements of intervention: 5 trials $(38.5 \%)$ included structure assessment as an intervention element; $28,31,33,34,388$ studies $(61.5 \%)$ provided predischarge education and training; ${ }^{27,28,31,33,34,38} 2$ studies $(15.3 \%)$ consisted of a structured care plan;27,28 7 trials $(53.8 \%)$ involved a home visit program; ${ }^{28,29,34,35,37-39} 8$ studies $(61.5 \%)$ included telephone follow-up;28,31-36,38 and 5 studies (38.4\%) encompassed a postdischarge educational program. ${ }^{30,34,36,38,39}$ Six trials (46.1\%) comprised other follow-up methods and extended interventions, such as web-based applications, ${ }^{27,28}$ educational booklets, ${ }^{37}$ telemonitoring, ${ }^{35}$ 24-h access to respiratory nurses and outpatient arrangements, ${ }^{33,34}$ and structured exercise and supervision programs. ${ }^{30}$

The median intervention duration was 3 months (range, $2-14$ months). The duration of intervention was $\leq 6$ months in 8 studies $(61.5 \%)$ and $>6$ months in 6 trials $(38.5 \%)$. Intervention providers were classified into a single profession $(38.5 \%)$ or multiple professions (ie, multifaceted interventions; $61.5 \%$ ). Most trials compared transitional care with usual care. Notably, one trial compared early discharge with home treatment supported by respiratory nurses and conventional treatments with hospital management for patient exacerbations of COPD. ${ }^{29}$ Another trial ${ }^{37}$ evaluated the effects of home-managed support on hospital readmission as compared with usual care (ie, standard hospital admission). 
Transitional Care, Readmissions, and Mortality in COPD

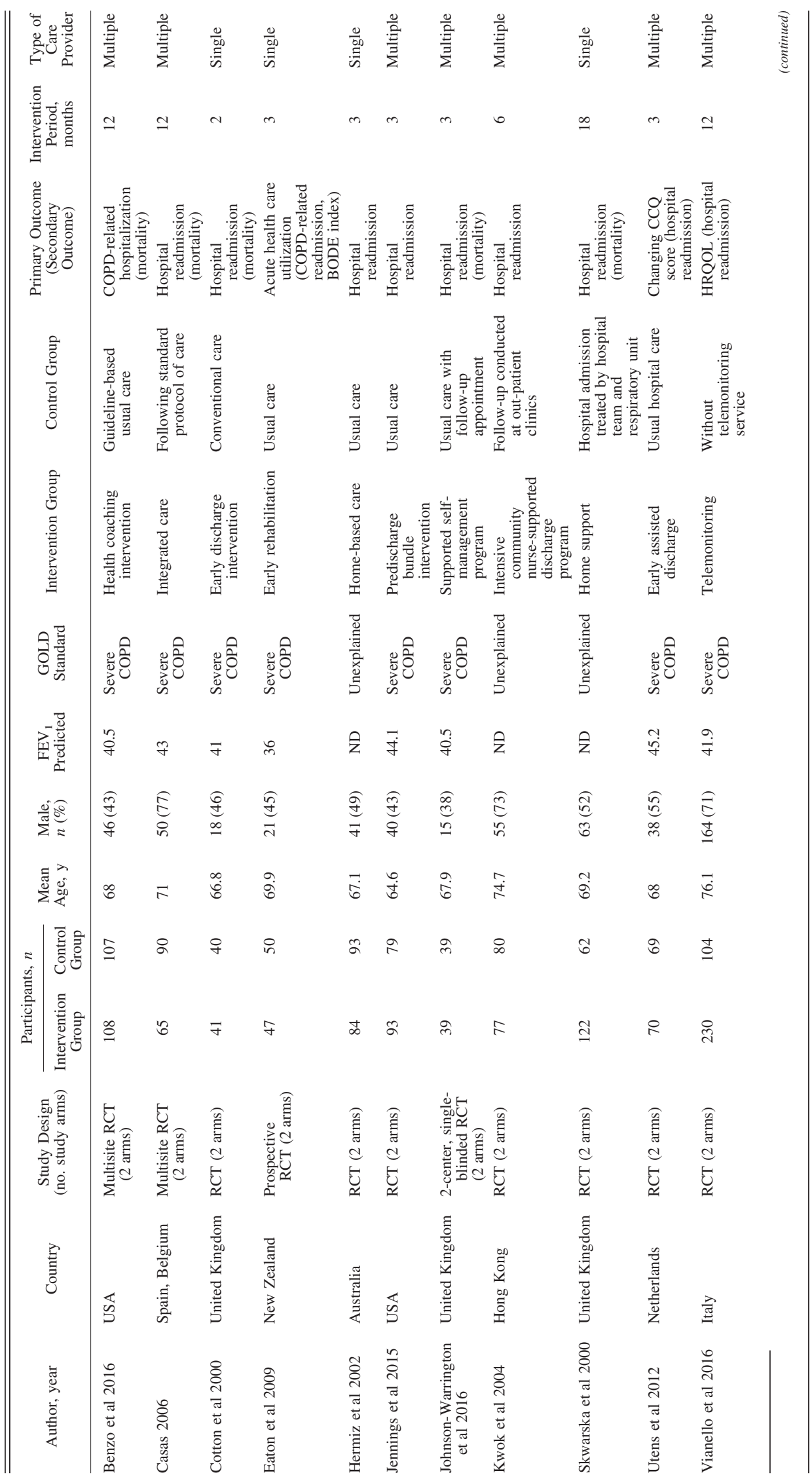


Transitional Care, Readmissions, and Mortality in COPD

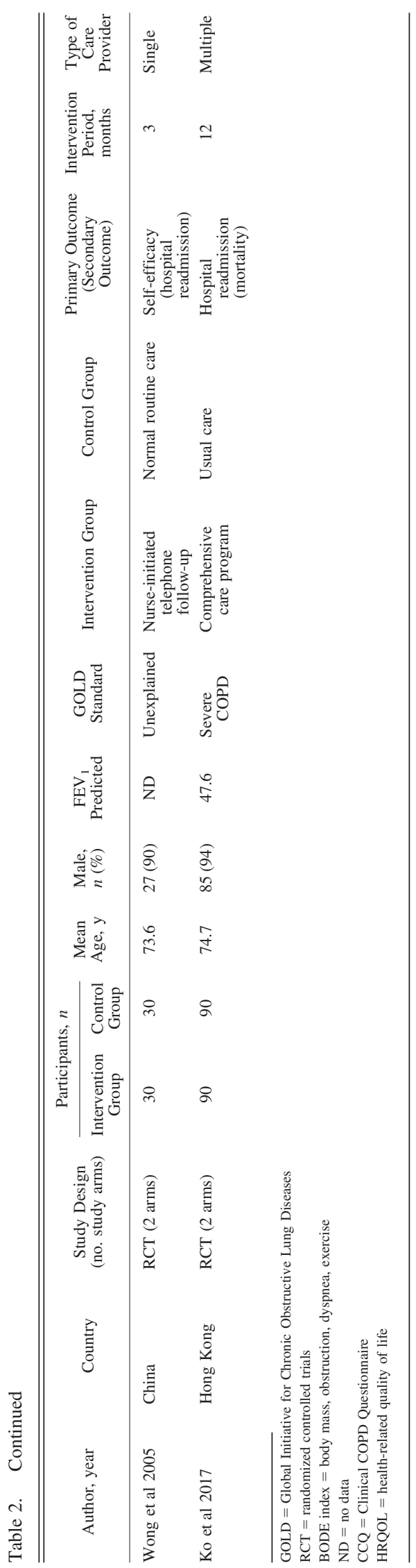

\section{Pooled Effects}

Figure 2 presents the pooled effect of transitional care on all-cause hospital readmission from 10 included trials $(\mathrm{OR}=0.720,95 \% \mathrm{CI} 0.531-0.978, P=.035)$. This finding indicated that transitional care had a significant effect on reducing all-cause hospital readmissions.

Four studies provided the data on the events of COPDrelated hospital readmission (Fig. 3). The pooled OR was 0.599 (95\% CI 0.421-0.852, $P=.004$ ), indicating a significant effect of transitional care on reducing the odds of COPD-related hospital readmissions.

The effects of transitional care on mortality rate were presented from 6 studies that provided data on the allcause mortality (Fig. 4). There were 1,117 subjects with COPD from the 6 included studies (pooled OR $=0.863$, $95 \%$ CI $0.576-1.294, P=.48$ ). This finding indicated that the effect of transitional care interventions on mortality rate was not statistically significant.

\section{Publication Bias}

Results from both the Begg rank correlation and the Egger regression test did not reveal significant publication bias among the studies assessing COPD-related hospital readmission $(P=.73$ and $P=.54$, respectively), among those assessing all-cause hospital readmissions $(P=.85$ and $P=.84$, respectively), and among those assessing mortality $(P=.060$ and $P=.15$, respectively $)$.

\section{Moderator Analyses}

We conducted moderator analyses to determine the effect of 3 dichotomous moderator variables on the relationship between transitional care and readmission. Table 3 presented results of all included trials with outcomes on either COPD-related or all-cause readmissions. Eight studies had intervention duration of $\leq 6$ months or shorter, and 6 studies had an intervention duration of $>6$ months. The pooled ORs of the group with intervention duration of $\leq 6$ months or shorter and that of the group with intervention duration of $>6$ months were $0.930(P=.63)$ and $0.531(P<.001)$, respectively; the point estimates between the 2 groups were significantly different $(P<.001)$.

In 9 studies, ${ }^{27,28,31,32,34-36,38}$ interventions were provided by multidisciplinary teams, whereas in 5 studies $29,30,36,38,39$ the interventions were delivered by care providers of a single profession. The pooled ORs were 0.693 for studies using multiple professions (95\% CI $0.519-0.925, P=.01$ ) and 0.667 for studies used a single profession $(95 \% \mathrm{CI}$ $0.427-1.043, P=.07)$; the pooled ORs between the 2 groups were significantly different $(P=.002)$.

Eight studies $28,31-34,38$ included telephone follow-up, and 6 studies $27,29,30,37,39$ did not include telephone follow-up as 


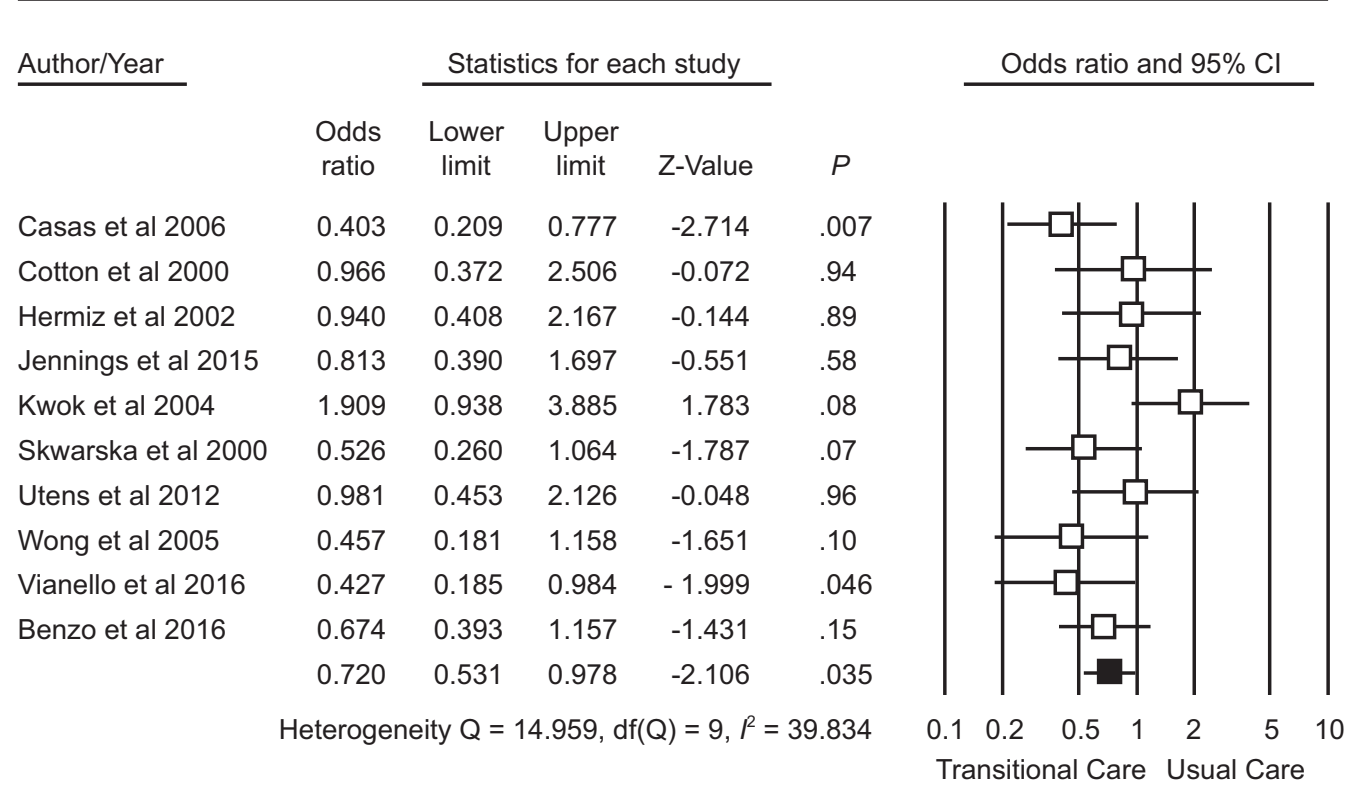

Fig. 2. Forest plot of effect sizes and pooled odds ratios of transitional care intervention for trials measuring all-cause hospital readmission in subjects with COPD using a random-effect model. Data on the events of all-cause hospital readmission were obtained from 10 trials. The black square represents the summary statistic, and lines show $95 \% \mathrm{Cl}$. A heterogeneity test on each subgroup's analysis showed no evidence of heterogeneity among studies.

Effect of Transitional Care on COPD-Related Readmission

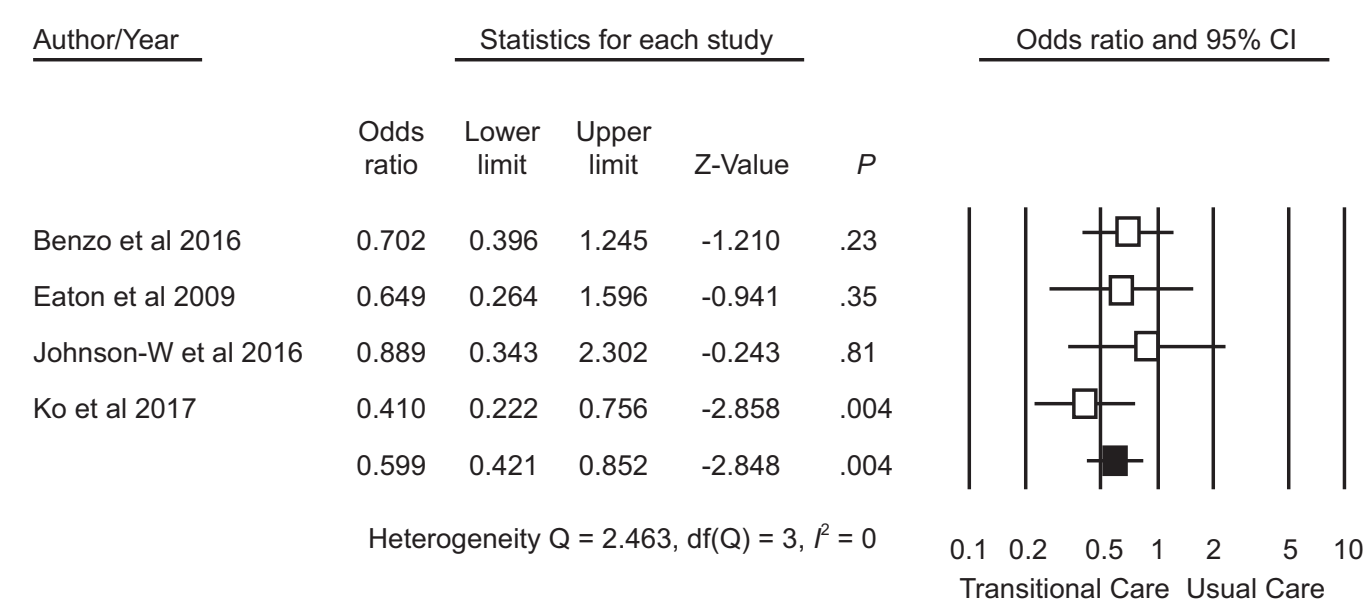

Fig. 3. Forest plot of effect sizes and pooled odds ratios of transitional care intervention for trials measuring COPD-related readmission in subjects with COPD using a random-effect model. Data on the events of COPD-related hospital readmission were obtained from 10 trials. The black square represents the summary statistic, and the lines show $95 \% \mathrm{Cl}$. A heterogeneity test on each subgroup's analysis showed no evidence of heterogeneity among studies.

an intervention element. The point estimates were 0.668 for studies using telephone follow-up (95\% CI $0.482-$ $0.926, P=.02$ ) and 0.707 for trials not including telephone follow-up (95\% CI 0.491-1.018, $P=.062$ ); the point estimates were significantly different between the 2 groups $(P=.002)$.
The pooled results of moderator analysis, which included 10 studies with the outcome of all-cause hospital readmissions, indicated a significant effect of duration intervention in moderating the association between transitional care and readmission. The pooled OR was 0.982 (95\% CI $0.705-1.367, P=.91)$ for studies with an inter- 


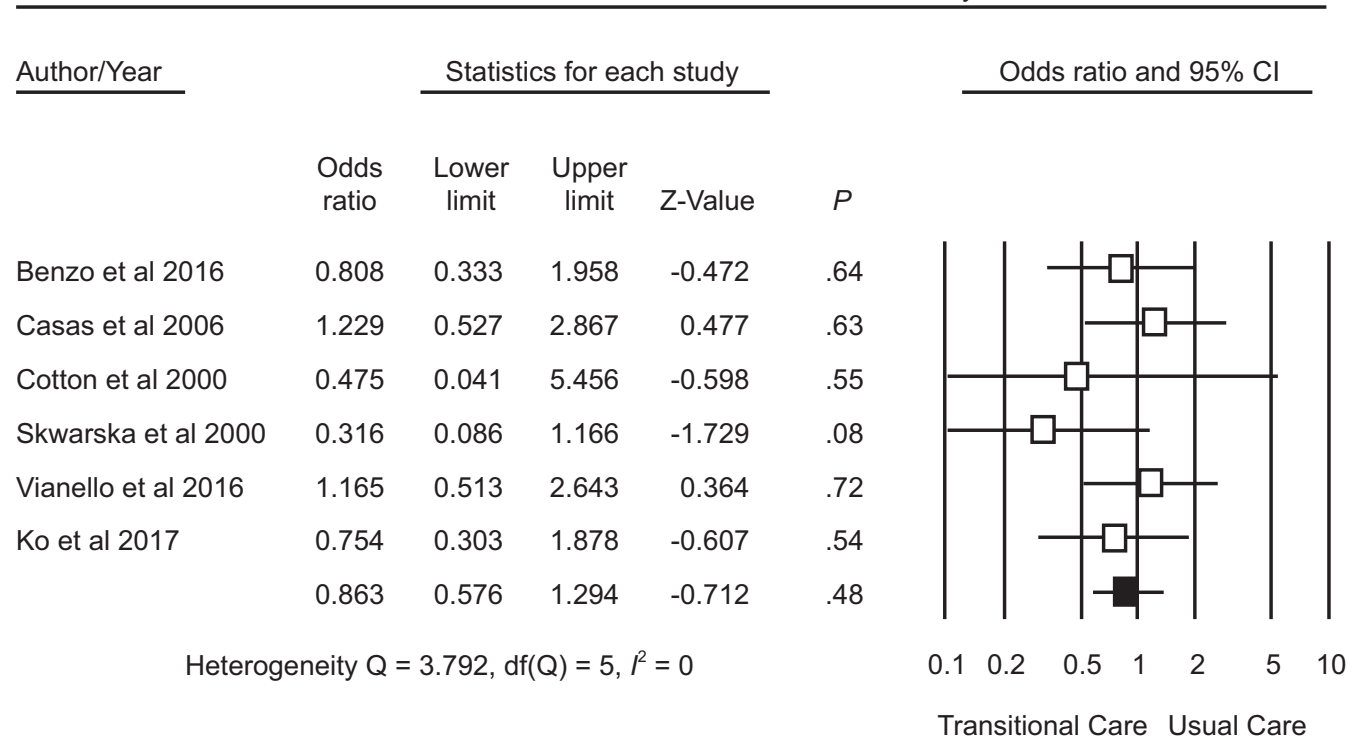

Fig. 4. Forest plot of effect sizes and pooled odds ratios of transitional care intervention for trials measuring all-cause mortality rate in subjects with COPD using a random-effect model. 6 studies provided data on the events of mortality. The black square represents the summary statistic, and lines show $95 \% \mathrm{Cl}$ among homogenous studies.

Table 3. Results of Moderator Analyses on All-Cause and COPD-Related Hospital Readmission

\begin{tabular}{|c|c|c|c|c|}
\hline Analysis & Trials, no.* & Odds Ratio (95\% CI) & $I^{2}$ & $P$ \\
\hline Duration of Intervention & & & & $<.001$ \\
\hline$\leq 6$ months & 8 & $0.930(0.693-1.249)$ & 0 & \\
\hline$>6$ months & 6 & $0.531(0.410-0.688)$ & 0 & \\
\hline Type of Care Provider & & & & .002 \\
\hline Single & 5 & $0.667(0.427-1.043)$ & 0 & \\
\hline Multiple & 9 & $0.693(0.519-0.925)$ & 49.306 & \\
\hline Telephone Follow-up & & & & .002 \\
\hline Yes & 8 & $0.668(0.482-0.926)$ & 57.382 & \\
\hline No & 6 & $0.707(0.491-1.018)$ & 0 & \\
\hline
\end{tabular}

The table shows moderating effects of 3 variables consisting of intervention, type of care providers, and telephone follow-up for the effects of transitional care on hospital readmission using randomeffect analysis. The $P$ values represent between-group significance,

* Represent one study provided data in the all-cause and COPD-related readmission.

vention duration of $\leq 6$ months, whereas the pooled OR for studies with an intervention duration of $>6$ months was 0.532 (95\% CI 0.373-0.734, $P<.001)$; the point estimates between the 2 groups were significantly different $(P=.007)$. However, the type of care providers and the use of telephone follow-up did not moderate the relationship between transitional care and all-cause hospital readmissions significantly $(P=.051$ and $P=.054$, respectively). Moreover, the findings of a meta regression analysis, performed with age and sex as predictors, revealed that age and percentage of males were not significantly associated with the effect size of transitional care for hospital readmissions $(P=.25$ and $P=.068$, respectively).

\section{Discussion}

Our meta-analysis examined the effect of transitional care interventions on hospital readmissions and mortality in subjects with COPD. We identified 13 trials that provided data on outcomes of interest for 2,029 subjects. Our findings indicate a significant effect of transitional care on reducing all-cause hospital readmissions and COPD-related readmissions in subjects with COPD. The odds of all-cause and COPD-related readmissions for those who received transitional care interventions decreased by $28 \%$ and $44 \%$, respectively. Our findings also indicate that transitional care interventions 
were not significantly associated with reduced all-cause mortality rate.

Although characteristics of transitional care interventions vary considerably across different systems of care, our findings support the valuable role of a transitional care approach in reducing frequent use of health care services by patients with COPD. The progression of COPD varies widely among patients; some patients have stable courses of progress, whereas others have persistent episodes of breathlessness, frequent acute relapses, and respiratory failure. ${ }^{40}$ Worsening airway function and COPD symptoms can be triggered by infection or air pollution. The triggers are preventable; however, failure to prevent them can lead to advanced disease severity, morbidity, negative effects on health-related quality of life, and mortality. ${ }^{6,7,9}$ These can account significantly for the consumption of health care resources. ${ }^{41}$ The worsening of COPD symptoms is related to frequent use of health care services; thus, discharge management to improve continuity of care has a role in eliminating the triggers of disease progression. A review indicated that when patients with COPD endure ongoing hospital treatments or emergency visits caused by exacerbation, care continuity is crucial in maintaining fragmented treatment regimens and providing necessary responses to any changes in a patient's respiratory system or prognosis. ${ }^{42}$ Furthermore, our findings concur with several metaanalyses showing the importance of transitional care interventions as programs with clinically meaningful effects in reducing the odds of patient readmissions and emergency visits in populations with heart failure ${ }^{43,44}$ Similarly, a previous study showed that a transitional stroke program reduced the odds of $30-\mathrm{d}$ readmission by $48 \% .45$ Our findings suggest the importance of transitional care as a tool to reduce hospital readmissions for patients with COPD.

Our moderator analyses revealed the significant effects of the care provider type, intervention duration, and telephone follow-up on the relationship between transitional care and hospital readmission. This finding can provide additional explanations regarding factors that may modify the success of the transitional care intervention in reducing hospital readmissions in patients with COPD, even though evidence on these elements of interventions may highlight some inconsistencies. Our findings concur with those from previous studies ${ }^{46,47}$ that a multidisciplinary team intervention may decrease $30-\mathrm{d}$ readmission rates and other forms of hospitalizations compared with usual care. In addition, the narrative analysis conducted by Hickman et $\mathrm{al}^{48}$ supported the notion that a multidisciplinary team underpinned with clear communication strategies can reduce readmission rates, mortality, and functional decline of older people. Furthermore, to some extent, the use of telephone follow-up provides beneficial effects on transitional care. Although Mistiaen and Poot $^{49}$ presented inconclusive evidence of the effectiveness of telephone in- terventions for managing postdischarge problems, other studies reported telephone follow-up as low-cost, feasible, and no-harm interventions. ${ }^{50-52}$ Thus, the transitional care intervention has a greater effect on reducing hospital readmission when the intervention lasted for $>6$ months, was provided by a multidisciplinary team, or incorporated telephone follow-up as an intervention element.

This meta-analysis found that transitional care interventions were ineffective in reducing mortality rates in subjects with COPD. In all included trials, one study reported a significant effect of transitional care for COPD-specific mortality, whereas other trials failed to support a beneficial effect of transitional care on all-cause mortality. The elements of transitional care interventions may require adjustments to more effectively address the mortality risk. Mortality risk factors for COPD are associated with acute relapse events and depend on the synergistic effect of predictive factors as well as the result of systematic care and prognosis effects of exacerbation. ${ }^{6}$ However, this finding should be interpreted with caution because it suggests that specific elements of transitional care interventions may be required to reduce mortality rates among patients with COPD.

This study has several limitations. First, heterogeneity across studies might have arisen from differences in intervention designs and assessment tools. Moderator analyses were likely insufficient for exploring whether intervention duration, care provider type, and telephone follow-up reduced mortality rates because the numbers of such studies were limited. Second, the results should be interpreted with caution because many included trials were subject to selection, performance, and detection bias as a result of ineffective concealment or lack of blinding to participants, research personnel, and outcome assessors.

\section{Conclusions}

Transitional care can be an effective approach for addressing the high number of hospital readmissions of patients with COPD. However, evidence of the beneficial effect of transitional care on the mortality rate of patients with COPD remains insufficient. The effects of transitional care interventions on hospital readmissions are moderated by the intervention duration, multidisciplinary approach, and use of telephone follow-up. Because patients with COPD vary in disease conditions, in risks of being readmitted to the hospital, and in risks of mortality, it is important to tailor transitional care interventions according to disease-related factors.

\section{REFERENCES}

1. World Health Organization. Global surveillance, prevention and control of chronic respiratory diseases: a comprehensive approach. Geneva, Switzerland: WHO Press, 2007. 
2. Global Initiative for Chronic Obstructive Lung Disease. GOLD 2017 Global Strategy for the Diagnosis, Management and Prevention of COPD. Available at: https://goldcopd.org/gold-2017-global-strategydiagnosis-management-prevention-copd. Accessed July 22, 2019.

3. MacNee W. Pathology, pathogenesis, and pathophysiology. BMJ 2006;332(7551):1202-1204.

4. El Wahsh RA, Ahmed MK, Yaseen RI. Evaluation of left ventricular function in patients with chronic obstructive pulmonary disease with or without pulmonary hypertension. Egypt J Chest Dis Tubercul 2013;62(4):575-582.

5. Shujaat A, Minkin R, Eden E. Pulmonary hypertension and chronic cor pulmonale in COPD. Int J Chron Obstruct Pulmon Dis 2007; 2(3):273-282.

6. Pavord ID, Jones PW, Burgel P-R, Rabe KF. Exacerbations of COPD. Int J Chron Obstruct Pulmon Dis 2016;11(Spec Iss):21-30.

7. Wedzicha JA, Donaldson GC. Exacerbations of chronic obstructive pulmonary disease. Respir Care 2003;48(12):1204-1213; discussion 1213-1205.

8. Chuang C. Transition of patients with COPD across different care settings: challenges and opportunities for hospitalists. Hosp Pract (1995) 2012;40(1):176-185.

9. Donaldson GC, Wedzicha JA. COPD exacerbations. 1: epidemiology. Thorax 2006;61(2):164-168.

10. Lawati N, Fitzgerald J. Acute exacerbation of chronic obstructive pulmonary disease. BC Med J 2008;50(3):138-142.

11. OHTAC. Chronic obstructive pulmonary disease (COPD) evidentiary framework. Ontario Health Technology Assessment Series 2012; 12(2):1-97.

12. Shah T, Churpek MM, Coca Perraillon M, Konetzka RT. Understanding why patients with COPD get readmitted: a large national study to delineate the Medicare population for the readmissions penalty expansion. Chest 2015;147(5):1219-1226.

13. Baxter N, McMillan V, Holzhauer-Barrie J, Robinson S, Stone P, Quint J, et al. Planning for every breath. National chronic obstructive pulmonary disease (COPD) audit programme: primary care audit (Wales) 2015-17. Data analysis and methodology. London: Royal College of Physicians, 2017.

14. Sharif R, Parekh TM, Pierson KS, Kuo Y-F, Sharma G. Predictors of early readmission among patients 40 to 64 years of age hospitalized for chronic obstructive pulmonary disease. Ann Am Thorac Soc 2014;11(5):685-694.

15. Simmering JE, Polgreen LA, Comellas AP, Cavanaugh JE, Polgreen PM. Identifying patients with COPD at high risk of readmission. Chronic Obstr Pulm Dis 2016;3(4):729-738.

16. Hunter LC, Lee RJ, Butcher I, Weir CJ, Fischbacher CM, McAllister $\mathrm{D}$, et al. Patient characteristics associated with risk of first hospital admission and readmission for acute exacerbation of chronic obstructive pulmonary disease (COPD) following primary care COPD diagnosis: a cohort study using linked electronic patient records. BMJ Open 2016;6(1):e009121.

17. Elixhauser A, Au DH, Podulka J. Readmission for chronic obstructive pulmonary disease, 2008: Statistical Brief 121. Healthcare Cost and Utilization Project. Available at: https://www.ncbi.nlm.nih.gov/ books/NBK65392. Accessed July 22, 2019.

18. Camargo L, Castellano MVO, Ferreira FC, Faria FV, Carvas N Jr. Hospitalization due to exacerbation of COPD: "real-life" outcomes. Rev Assoc Med Bras (1992) 2017;63(6):543-549.

19. Duffy S, Barnett S, Civic B, Mamary AJ, Criner GJ. Risk of death by comorbidity prompting rehospitalization following the initial COPD hospitalization. Chronic Obstr Pulm Dis 2015;2(1):17-22.

20. Naylor M, Keating SA. Transitional care. Am J Nurs 2008;108 (9 Suppl):58-63.
21. Coleman EA, Boult C. Improving the quality of transitional care for persons with complex care needs. J Am Geriatr Soc 2003;51(4):556557.

22. Comín-Colet J, Enjuanes C, Lupón J, Cainzos-Achirica M, Badosa N, Verdú JM. Transitions of care between acute and chronic heart failure: critical steps in the design of a multidisciplinary care model for the prevention of rehospitalization. Rev Esp Cardiol (Engl Ed) 2016;69(10):951-961.

23. Liu M, Zhang Y, Li D-D, Sun J. Transitional care interventions to reduce readmission in patients with chronic obstructive pulmonary disease: a meta-analysis of randomized controlled trials. Chinese Nurs Res 2017;4(2):84-91.

24. Moher D, Liberati A, Tetzlaff J, Altman DG. Preferred reporting items for systematic reviews and meta-analyses: the PRISMA statement. PLOS Med 2009;6(7):e1000097.

25. Borenstein M, Higgins JP, Hedges LV, Rothstein HR. Basics of meta-analysis: $\mathrm{I}(2)$ is not an absolute measure of heterogeneity. Res Synth Methods 2017;8(1):5-18.

26. Egger M, Smith GD, Schneider M, Minder C. Bias in meta-analysis detected by a simple, graphical test. BMJ 1997;315(7109):629-634.

27. Benzo R, Vickers K, Novotny P, Tucker S, Hoult J, Neuenfeldt P, et al. Health coaching and chronic obstructive pulmonary disease rehospitalization: a randomized study. Am J Respir Crit Care Med 2016;194(6):672-680.

28. Casas A. Integrated care prevents hospitalisations for exacerbations in COPD patients. Eur Respir J 2006;28(1):123-130.

29. Cotton M, Bucknall C, Dagg K, Johnson M, MacGregor G, Stewart $\mathrm{C}$, et al. Early discharge for patients with exacerbations of chronic obstructive pulmonary disease: a randomised controlled trial. Thorax 2000;55:902-906

30. Eaton T, Young P, Fergusson W, Moodie L, Zeng I, O'Kane F, et al. Does early pulmonary rehabilitation reduce acute health-care utilization in COPD patients admitted with an exacerbation? A randomized controlled study. Respirology 2009(14):230-238.

31. Jennings JH, Thavarajah K, Mendez MP, Eichenhorn M, Kvale P, Yessayan L. Predischarge bundle for patients with acute exacerbations of COPD to reduce readmissions and ED visits. Chest 2015; 147(5):1227-1234.

32. Johnson-Warrington V, Rees K, Gelder C, Morgan M, Singh S. Can a supported self-management program for COPD upon hospital discharge reduce readmissions? A randomized controlled trial. Int J Chron Obstruct Pulmon Dis 2016;11:1161-1169.

33. Ko FW, Cheung NK, Rainer TH, Lum C, Wong I, Hui DS. Comprehensive care programme for patients with chronic obstructive pulmonary disease: a randomised controlled trial. Thorax 2017;72(2): 122-128.

34. Utens CM, Goossens LM, Smeenk FW, Rutten-van Molken MP, van Vliet M, Braken MW, et al. Early assisted discharge with generic community nursing for chronic obstructive pulmonary disease exacerbations: results of a randomised controlled trial. BMJ Open 2012; 2(5): 001684

35. Vianello A, Fusello M, Gubian L, Rinaldo C, Dario C, Concas A, et al. Home telemonitoring for patients with acute exacerbation of chronic obstructive pulmonary disease: a randomized controlled trial. BMC Pulm Med 2016;16(1):157.

36. Wong K, Wong F, Chan M. Effects of nurse-initiated telephone follow-up on self-efficacy among patients with chronic obstructive pulmonary disease. J Adv Nurs 2005; 49(2):210-222.

37. Skwarska E, Cohen G, Skwarski K, Lamb C, Bushell D, Parker S, et al. Randomised controlled trial of supported discharge in patients with exacerbations of chronic obstructive pulmonary disease. Thorax 2000;55:907-912.

38. Kwok T, Lum C, Chan H, Ma H, Lee D, Woo J. A randomized, controlled trial of an intensive community nurse-supported discharge 
program in preventing hospital readmissions of older patients with chronic lung disease. J Am Geriatr Soc 2004;52(8):1240-1246.

39. Hermiz O, Comino E, Marks G, Daffurn K, Wilson S, Harris M. Randomised controlled trial of home based care of patients with chronic obstructive pulmonary disease. BMJ 2002;325:1-5.

40. Shaw JG, Vaughan A, Dent AG, O'Hare PE, Goh F, Bowman RV, et al. Biomarkers of progression of chronic obstructive pulmonary disease (COPD). J Thorac Dis 2014;6(11):1532-1547.

41. MacIntyre N, Huang YC. Acute exacerbations and respiratory failure in chronic obstructive pulmonary disease. Proc Am Thorac Soc 2008; 5(4):530-535.

42. Fromer L. Implementing chronic care for COPD: planned visits, care coordination, and patient empowerment for improved outcomes. Int J Chron Obstruct Pulmon Dis 2011;6:605-614.

43. Feltner C, Jones CD, Cene CW, Zheng ZJ, Sueta CA, CokerSchwimmer EJ, et al. Transitional care interventions to prevent readmissions for persons with heart failure: a systematic review and meta-analysis. Ann Intern Med 2014;160(11):774-784.

44. Vedel I, Khanassov V. Transitional care for patients with congestive heart failure: a systematic review and meta-analysis. Ann Fam Med 2015;13(6):562-571.

45. Condon C, Lycan S, Duncan P, Bushnell C. Reducing readmissions after stroke with a structured nurse practitioner/registered nurse transitional stroke program. Stroke 2016;47(6):1599-1604.
46. Stranges PM, Marshall VD, Walker PC, Hall KE, Griffith DK, Remington T. A multidisciplinary intervention for reducing readmissions among older adults in a patient-centered medical home. Am J Manag Care 2015;21(2):106-113.

47. Reidt SL, Holtan HS, Larson TA, Thompson B, Kerzner LJ, Salvatore TM, et al. Interprofessional collaboration to improve discharge from skilled nursing facility to home: preliminary data on postdischarge hospitalizations and emergency department visits. J Am Geriatr Soc 2016;64(9):1895-1899.

48. Hickman LD, Phillips JL, Newton PJ, Halcomb EJ, Al Abed N, Davidson PM. Multidisciplinary team interventions to optimise health outcomes for older people in acute care settings: a systematic review. Arch Gerontol Geriatr 2015;61(3):322-329.

49. Mistiaen P, Poot E. Telephone follow-up, initiated by a hospital-based health professional, for postdischarge problems in patients discharged from hospital to home. Cochrane Database Syst Rev 2006;(4):CD004510.

50. Crocker JB, Crocker JT, Greenwald JL. Telephone follow-up as a primary care intervention for postdischarge outcomes improvement: a systematic review. Am J Med 2012;125(9):915-921.

51. Menchine M, Oberfoell S, Schriger D, Walker C, Riddell J, Arora S. Improving telephone follow-up for patients discharged from the emergency department: results of a randomized controlled trial. Acad Emerg Med 2013;20(5):456-462.

52. Kirk C. Telephone follow-up of older people after hospital admissions. Curr Aging Sci 2014;7(2):144-153. 